研 究

\title{
Magnetic Response of a Single-Crystal Thin Film $\mathrm{Bi}_{2} \mathrm{Sr}_{2} \mathrm{CaCu}_{2} \mathrm{Oy}$
}

\author{
Shinjiro Tochihara, Ken-ichiro Harashima, Hiroshi Yasuoka and Hiromasa Mazaki
}

Department of Mathematics and Physics, National Defense Academy, 1-10-20 Hashirimizu, Yokosuka 239.

Received April 14, 1997

\section{SYNOPSIS}

To test the utility of the modified Kim-Anderson model for study of the magnetic response of ceramic superconductors, we measured ac complex susceptibilities $\chi_{n}=\chi_{n}{ }^{\prime}-i \chi_{n}{ }^{\prime}(n=1,2,3,5,7)$ of a single-crystal thin film $\mathrm{Bi}_{2} \mathrm{Sr}_{2} \mathrm{CaCu}_{2} \mathrm{O}_{y}$, and attempted to simulate the results in the framework of this model. Analyses of $\chi_{n}$ have shown that generation of the higher-harmonics can be reproduced pretty well, where the material parameters $k$ and $B_{0}$, the surface barrier $\Delta H$ and the lower critical field $H_{c l}$ involved were used as the fitting parameters. From the even- $n$ harmonic susceptibility $\chi_{2}$ which reflects the asymmetric nature of the magnetization curve, we foundthat the critical-current density $J_{c}$ of the $\mathrm{Bi}_{2} \mathrm{Sr}_{2} \mathrm{CaCu}_{2} \mathrm{O}_{y}$ film depends sensitively on the local flux density $B_{i}$. This result is a provoking contrast to the $B_{i}$ dependence of $J_{c}$ for an $\mathrm{YBa}_{2} \mathrm{Cu}_{3} \mathrm{O}_{y}$ film.

KEY WORDS

modified Kim-Anderson model, surface barriers, single-crystal thin film, harmonic susceptibility.

\section{Introduction}

The Kim-Anderson model introduced by Kim, Hempstead and Strnad" and Anderson ${ }^{2)}$ has been widely used for study of the magnetic property of type-II superconductors. The essential aspect of this model is that, when a magnetic field is applied to a sample, a macroscopic supercurrent circulates in the sample with a critical-current density $J_{c}\left(B_{i}\right)$, where $B_{i}$ is the local flux density inside the specimen. They assumed that the critical-current density has the form

$$
J_{c}=k \lambda\left(B_{0}+\left|B_{i}\right|\right)
$$

where $k$ and $B_{0}$ are material parameters. As pointed out by Chen and Goldfarb ${ }^{3)}$, the relation given by Eq.(1) is a generalized form of the critical-state model, because it leads either to the liner mode ${ }^{4)}$ or to the Bean mode $^{5)}$ or to the power-law model ${ }^{6)}$, depending on the relative magnitude of $k, B_{0}$ and $B_{i}$.

To test the utility of the Kim-Anderson model ${ }^{1,2)}$ through ac complex susceptibilities as a probe, we have carried out a series of investigations, where we used several kinds of ceramic superconductors, $\mathrm{YBa}_{2} \mathrm{Cu}_{3} \mathrm{O}_{\mathrm{y}}(\mathrm{YBCO})$ single-crystal thin films ${ }^{7}$, an $\mathrm{Fe}$ doped $\mathrm{YBCO}$ sintered sample ${ }^{8)}$, a melt-processed $\mathrm{YBCO}^{9)}, \mathrm{HoBa}_{2} \mathrm{Cu}_{4} \mathrm{O}_{\mathrm{y}}{ }^{(0)}$ and $\mathrm{Bi}_{1.6} \mathrm{~Pb}_{0.4} \mathrm{Sr}_{2} \mathrm{Ca}_{2} \mathrm{Cu}_{3} \mathrm{O}_{\mathrm{y}}{ }^{(1)}$.
In these studies, the observed complex susceptibilities as a function of temperature were analyzed using the magnetization equations ${ }^{12)}$ based on the KimAnderson model. The reproducibility of the data has been found to be pretty good, except in one case of single-crystal thin films. In these previous treatments, we have neither taken into consideration surface barriers $\Delta H$ nor lower critical fields $H_{c 1}$, both of these were assigned to be zero.

According to Dunn and Hlawiczka ${ }^{13)}$, the magnetization curves are somewhat complicated due to the nonzero values of $\Delta H$ and $H_{c 1}$, but the effective field in the sample may be described by the following expression ${ }^{13)}$,

$$
H_{\text {eff }}=H-\frac{H}{|H|} H_{c l}-\frac{d H / d t}{|d H / d t|} \Delta H,
$$

where $H$ is the applied field, $\Delta H$ and $H_{c 1}$ are assumed to be constants.

For analyses of $\chi_{n}$ for the thin film which cannot be reproduced by the Kim-Anderson model, we modified the Bean model using $H_{e f f}$ instead of $H$ and fully derived the magnetization equations for type-II superconductors $^{14)}$. Applying these equations, we could qualitatively well reproduce $\chi_{n}$ for YBCO 
single-crystal thin films ${ }^{15}$.

Recently, we extended the modified Bean model to a more generalized case, i. e., we modified the KimAnderson model by using $H_{e f f}$ and fully derived the magnetization equations for an infinitely long cylinder. The theoretical derivation will appear elsewhere.

The time-dependent magnetization $M(\omega t)$ of a specimen for a magnetic field $H(\omega t)=H_{\mathrm{dc}}+H_{\mathrm{ac}} \cos (\omega t)$ can generally be expressed in the form of a Fourier expression:

$$
M(\omega t)=\chi_{0} H_{\mathrm{dc}}+H_{\mathrm{ac}} \sum_{n=1}^{\infty} \operatorname{Re}\left[\chi_{n} \exp (\operatorname{in} \omega t)\right]
$$

where $\operatorname{Re}[$ ] denotes the real part of a complex variable and $\omega=2 \pi f$, where $f$ is the fundamental frequency. The first term $\chi_{0} H_{\mathrm{dc}}$ is an offset by a dc magnet field $H_{\mathrm{dc}}$, and the second is the ac component which varies in time with the ac magnetic field $H_{\mathrm{ac}} \cos (\omega t)$. Here we define $\chi_{n} \equiv \chi_{n}{ }^{\prime}-i \chi_{n}{ }^{\prime}(n=1,2, \ldots)$ as the Fourier coefficients of the magnetization, where $\chi_{1}$ is the fundamental susceptibility and the others are the higher-harmonic susceptibilities.

In this paper, we present the observed $\chi_{n}$ of a singlecrystal thin film $\mathrm{Bi}_{2} \mathrm{Sr}_{2} \mathrm{CaCu}_{2} \mathrm{O}_{\mathrm{y}}(1 \mathrm{~cm} \times 1 \mathrm{~cm} \times 400 \mathrm{~A})$. Analyses of the data were carried out using the modified Kim-Anderson model. Some discussion of the magnetic response of the film is also given.

\section{Experimental}

\subsection{Sample preparation}

A single-crystal thin film $\mathrm{Bi}_{2} \mathrm{Sr}_{2} \mathrm{CaCu}_{2} \mathrm{O}_{y}$ was prepared by the RF magnetron spattering method. The film epitaxially grew with the [0 011$]$ axis normal to the $\left(\begin{array}{lll}1 & 0 & 0\end{array}\right)$ surface of the $\mathrm{MgO}$ single crystal. The phase identification was made by $\mathrm{X}$-ray diffraction patterns using $\mathrm{Cu}-\mathrm{Ka}$ radiations. The data have only $(00 l)(l=1,2, \ldots)$ reflections of $\mathrm{Bi}_{2} \mathrm{Sr}_{2} \mathrm{CaCu}_{2} \mathrm{O}_{y}$ structure, suggesting that this sample is a single crystal.

\subsection{Measurement of $\chi_{n}$}

The measuring system of $\chi_{1}$ consisted of the Hartshorn bridge and the temperature control system. To avoid the bridge imbalance during one run of the experiment, the sample coil was directly immersed in a liquid He bath. A null adjustment of the bridge was made at $100 \mathrm{~K}$ and the sample temperature was decreased down to $4.2 \mathrm{~K}$ at a rate of about $0.3 \mathrm{~K} / \mathrm{min}$. Phase setting of the lock-in amplifier was carefully made so as to give variation only to the in-phase signals, against the change in the bridge inductance. The ac magnetic field $H_{\mathrm{ac}} \cos (\omega t)$ was applied to the sample using a function generator, where the applied ac field is parallel to the $c$ axis.

For measurements of higher-harmonic susceptibilities $\chi_{n}(n=2,3,5,7)$, the device was modified, where two function generators were used. The ac magnetic field was applied using one generator $F 1$ and the other generator F2 was used to supply the $n$ th-harmonic sinusoidal wave synchronized with F1. In this experiment, magnetic shielding was not used, and so the applied ac field was superimposed on the Earth's field. Using a gaussmeter, the Earth's field was determined to be $\mu_{0} H_{\mathrm{dc}}=0.03 \mathrm{mT}$, where $\mu_{0}=4 \pi \times 10^{-7} \mathrm{H} / \mathrm{m}$. Details of the measuring system were previously reported ${ }^{16)}$.

\section{Results and discussion}

In Fig.1, we show the real and imaginary components of $\chi_{1}$ versus temperature. The applied field amplitude $\mu_{0} H_{\mathrm{ac}}=0.01 \mathrm{mT}$ and $f=132 \mathrm{~Hz}$. The onset temperature $T_{c}$ (onset) is $78.0 \mathrm{~K}$.

In Fig.2, we show the $\chi_{n}-t_{r}(n=2,3,5,7)$ curves under $\mu_{0} H_{\mathrm{ac}}=0.1 \mathrm{mT}$ and $f=132 \mathrm{~Hz}$, where $t_{r}\left(\equiv T / T_{c}\right)$ is the reduced temperature. The specific features are

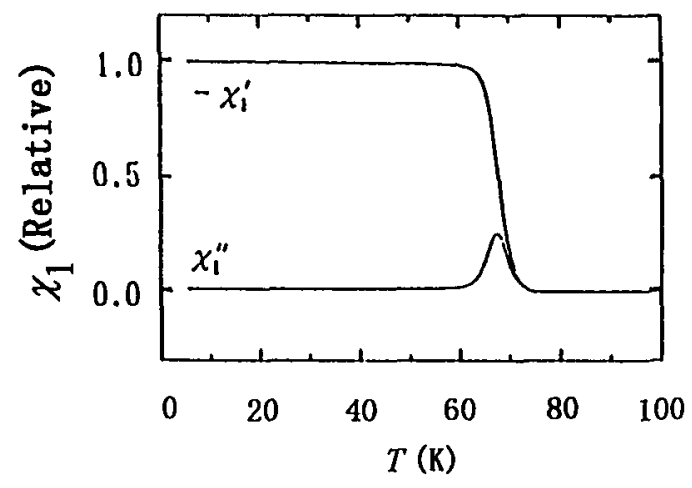

Fig.1 Real and imaginary components of the fundamental susceptibility $\chi_{1}$ vs temperature $T$ for a single-crystal thin film $\mathrm{Bi}_{2} \mathrm{Sr}_{2} \mathrm{CaCu}_{2} \mathrm{Oy}$, where $\mu_{0} H_{\mathrm{ac}}=0.01 \mathrm{mT}$ and $f=132 \mathrm{~Hz}$. 
followings:

(1) The values of $\chi_{2}$ are very large in comparison with those of YBCO single-crystal thin films ${ }^{15}$.

(2) In the $\chi_{5}-t_{r}$ and $\chi_{7}-t_{r}$ curves, we find small oscillations in the lower temperature region $\left(t_{r}<\sim\right.$ 0.85 ).

When the sample magnetization $M(\omega t)$ is expressed by Eq.(3), the real and imaginary components of $\chi_{n}$ can be calculated by

$$
\begin{aligned}
& \chi_{n}^{\prime}=\frac{1}{\pi H_{\mathrm{ac}}} \int_{0}^{2 \pi} M(\omega t) \cos (\omega t) d(\omega t), \\
& \chi_{n}^{\prime \prime}=\frac{1}{\pi H_{\mathrm{ac}}} \int_{0}^{2 \pi} M(\omega t) \sin (\omega t) d(\omega t),
\end{aligned}
$$

Using the magnetization equations derived from the modified Kim-Anderson model, we evaluated $M$. To

$$
\left[\times 10^{-3}\right]
$$

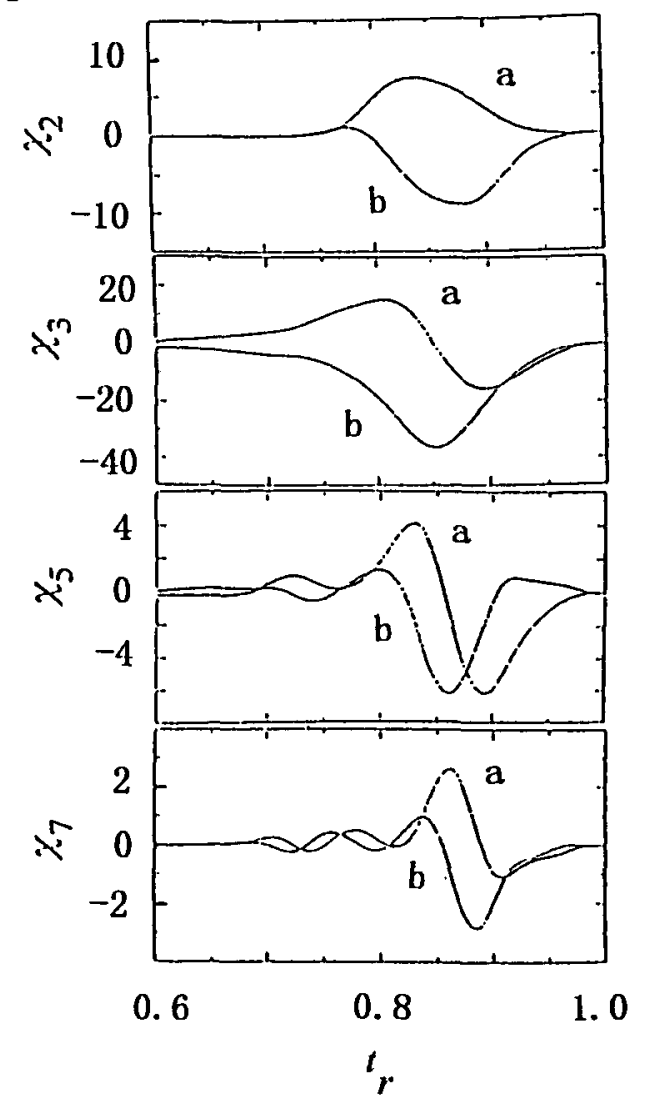

Fig. 2 Observed $\chi_{2}, \chi_{3}, \chi_{5}, \chi_{7}$ vs $t_{r}\left(\equiv T / T_{c}\right)$ for a single-crystal thin film $\mathrm{Bi}_{2} \mathrm{Sr}_{2} \mathrm{CaCu}_{2} \mathrm{O}_{\mathrm{y}}$, where $\mu_{0} H_{\mathrm{ac}}=0.1 \mathrm{mT}$ and $f=132$ $\mathrm{Hz}$. (a) is the real component and (b) is the imaginary component. Data are normalized to $\chi_{1}$. carry out the evaluation, one period of the alternating magnetic field $(0 \leq \omega t \leq 2 \pi)$ was divided into 360 regular intervals, and $M(\omega t)$ was numerically obtained at each point. Using the calculated results of $M(\omega t)$ in Eqs.(4) and (5), we evaluated $\chi_{n}^{\prime}$ and $\chi_{n}$ ".

The temperature dependence of $\chi_{n}$ comes from the parameters which involve $T$. In the present case, the temperature dependence of $\chi_{n}$ is introduced through $k, B_{0}, \Delta H$ and $H_{c 1}$. Here we take $H_{c l}=0$. The physical reason for this assumption is discussed later. The temperature dependence of $k$ and $B_{0}$ were determined experimentally as $k\left(t_{r}\right)=k(0)\left(1-t_{r}\right)^{3}$ and $B_{0}\left(t_{r}\right)=B_{0}(0)$ $\left(1-t_{r}\right)^{3 / 2}$. Although the temperature dependence of $\Delta H$ still remains to be resolved, we tentatively assumed $\Delta H\left(t_{r}\right)=\Delta H(0)\left(1-t_{r}\right)^{3 / 2}$ to reproduce the

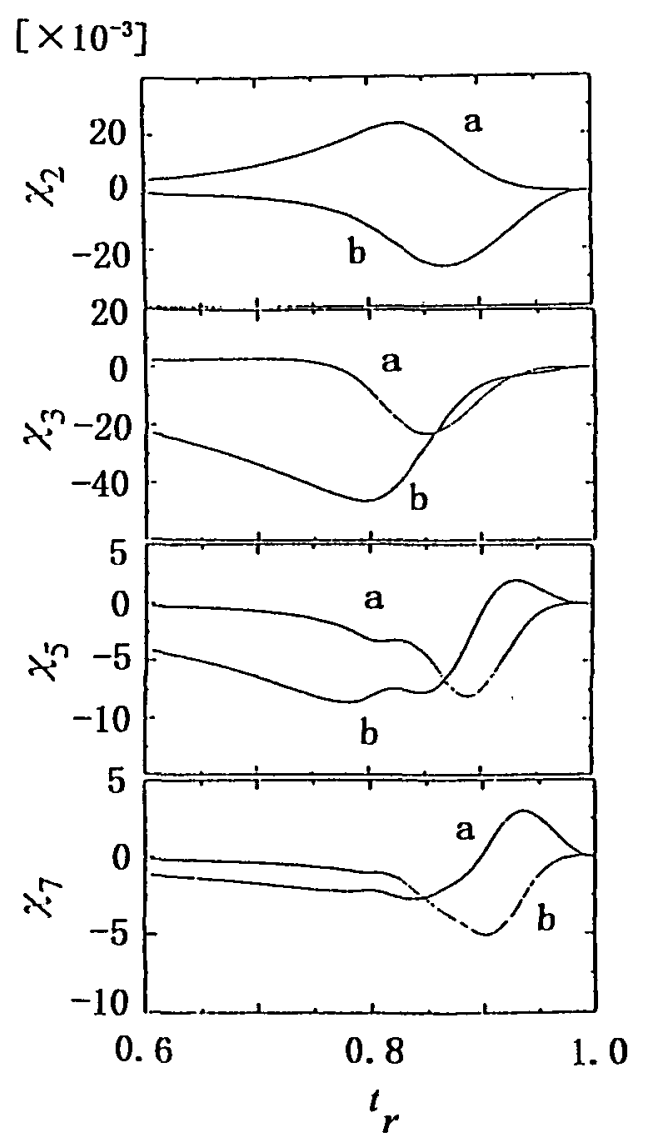

Fig.3 $\chi_{2}, \chi_{3}, \chi_{5}, \chi_{7}$ vs $t_{r}$ calculated by the Kim-Anderson model $\left(\Delta H=0\right.$ and $\left.H_{c 1}=0\right)$, where $k(0)=250 \mathrm{~N} / \mathrm{m}^{3}, B_{0}(0)=1$ $\mathrm{mT}, \mu_{0} H_{\mathrm{ac}}=0.1 \mathrm{mT}$ and $\mu_{0} H_{\mathrm{dc}}=0.03 \mathrm{mT}$. (a) is the real component and (b) is the imaginary component. 
profile of the observed $\chi_{n}-t_{r}$ curves. Here $k(0), B_{0}(0)$ and $\Delta H(0)$ are fitting parameters.

In Fig. 3 , we show calculated $\chi_{n}(n=2,3,5,7)$ by the original Kim-Anderson model. As seen in the figures, we can reproduce the magnetic behavior in the temperature region near $T_{c}\left(\sim 0.85<t_{r}<1\right)$, but small oscillations which appear in the temperature region $t_{r}<\sim 0.85$ of the $\chi_{5}-t_{r}$ and $\chi_{7}-t_{r}$ curves cannot be reproduced.

When we substitute $M(\omega t)$ derived from the modified Kim-Anderson model $(\Delta H \neq 0)$ into Eqs.(4) and (5), the small oscillations appeared in the observed $\chi_{5}-t_{r}$ and $\chi_{7}-t_{r}$ curves can be reproduced (see Fig.4). This fact strongly suggests that the surface barrier $\Delta H$ plays an essential role for the

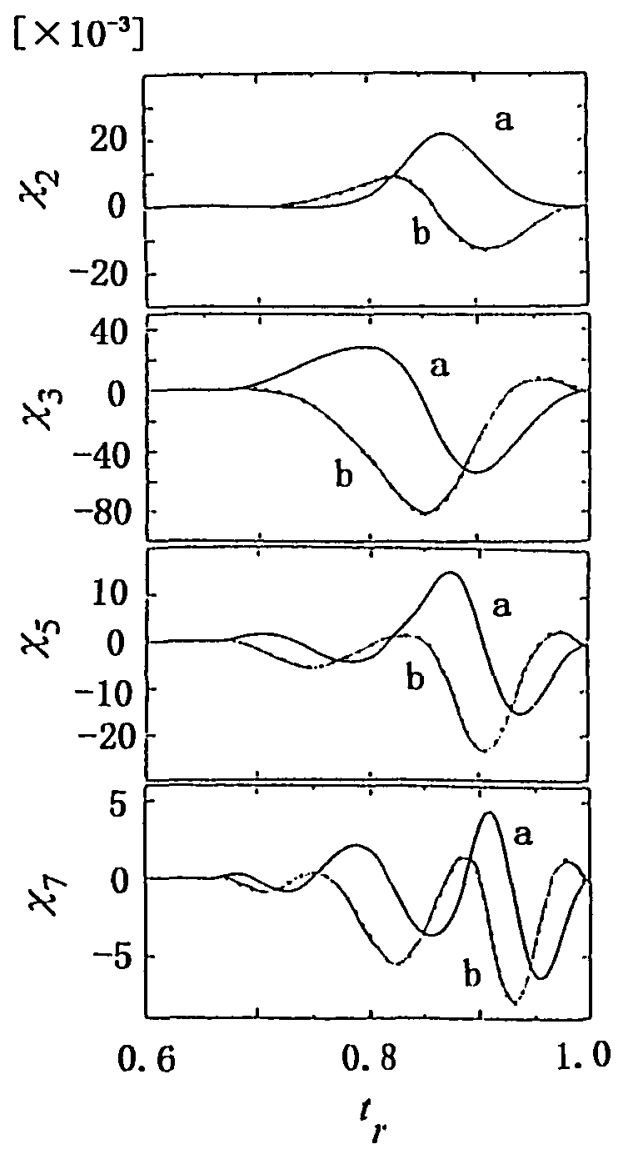

Fig.4 $\chi_{2}, \chi_{3}, \chi_{5}, \chi_{7}$ vs $t_{r}$ calculated by the modified KimAnderson model $\left(H_{\mathrm{cl}}=0\right)$, where

$k(0)=250 \mathrm{~N} / \mathrm{m}^{3}, B_{0}(0)=1 \mathrm{mT}, \mu_{0} \Delta H(0)=0.5 \mathrm{mT}, \mu_{0} H_{x}=0.1 \mathrm{mT}$ and $\mu_{0} H_{\mathrm{dc}}=0.03 \mathrm{mT}$. (a) is the real component and (b) is the imaginary component. magnetic response of the thin-film superconductor.

In the calculations of $\chi_{n}$ demonstrated in Fig.4, we assumed $H_{c 1}=0$. This assumption seems to be reasonable because the demagnetization effect for a thin film should be extremely large. Supposing a flat spheroid with a thin pancake shape whose length of the short axis reaches a limit value of zero, the demagnetization coefficient $N$ can be approximated by $N=1-\frac{1}{2} \pi(d / R)$, where $2 d$ is the film thickness and $R$ is the radius ${ }^{17}$. Eventually, for a thin film, $N$ is very close to 1 . This causes the increase of the internal field and thus the practical value of the lower critical field becomes zero.

As mentioned before, the even- $n$ harmonics appear only when the magnetization curve has an asymmetric feature. The nonzero values of $\chi_{2}$ shown in Fig.2 should therefore be originated from the asymmetric $M(H)$ curves. In Fig.5, we show the $M(H)$ curve used for theoretical simulation of $\chi_{2}$ given in Fig.4, where $k(0)=250 \mathrm{~N} / \mathrm{m}^{3}, B_{0}(0)=1 \mathrm{mT}, \mu_{0} \Delta H(0)=0.5 \mathrm{mT}$ and $H_{c 1}=0$. As demonstrated in Fig.5, the $M(H)$ curve has a strong asymmetric feature which is caused by the existence of $H_{\mathrm{dc}}$ and the $B_{i}$-dependent $J_{c}$, and this certainly gives rise to relatively large values of $\chi_{2}$.

It should be noted that when $J_{c}$ is a constant, the $M(H)$ loop tends to hold a symmetric nature even when $H_{\mathrm{dc}} \neq 0$. Since the dependence of $J_{c}$ on $B_{i}$ for YBCO thin films is quite small, $\chi_{2}$ for them is expected to be small. In fact, the previous observations of $\chi_{2}$ for YBCO thin films was relatively small and consequently the modified Bean model could reproduce qualitatively well the experimental $\chi_{2}{ }^{15)}$.

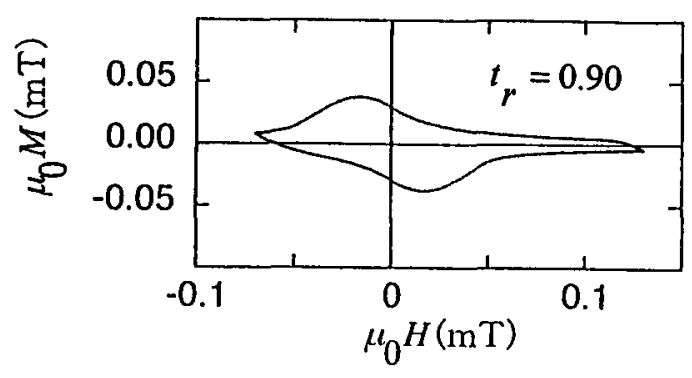

Fig.5 $M(H)$ loop at $t_{r}=0.90$ calculated by using the modified Kim-Anderson model. 


\section{Summary}

We measured the superconducting transition of a single-crystal thin film $\mathrm{Bi}_{2} \mathrm{Sr}_{2} \mathrm{CaCu}_{2} \mathrm{O}_{\mathrm{y}}$ in terms of higher-harmonic ac susceptibilities $\chi_{n}(n=2,3,5,7)$. Analyses of the results were made in the modified Kim-Anderson model which takes into consideration surface barriers $\Delta H$ and lower critical fields $H_{c l}$. Comparison of the observed $\chi_{n}$ with the calculated results has revealed that the modified Kim-Anderson model can reproduce pretty well the magnetic behavior of the present film. This means that the nonzero value of $\Delta H$ together with the $B_{i}$ dependence of $J_{c}$ plays an essential role for the magnetic response of the thin film $\mathrm{Bi}_{2} \mathrm{Sr}_{2} \mathrm{CaCu}_{2} \mathrm{O}_{\mathrm{y}}$.

\section{Acknowledgments}

We are grateful to Dr. M. Hirota of Technology Research and Development Institute of Japan Defense Agency for the sample preparation of a single-crystal thin film $\mathrm{Bi}_{2} \mathrm{Sr}_{2} \mathrm{CaCu}_{2} \mathrm{O}_{y}$.

\section{References}

1) Y.B.Kim, C.F.Hempstead and A.R.Strnad: Phys. Rev. Lett., 9(1962)306; Y.B.Kim, C.F. Hempstead and A.R.Strnad: Phys. Rev., 129 (1963) 528.

2) P.W.Anderson: Phys. Rev. Lett., 9(1962)309.

3) D.-X.Chen and R.B.Goldfarb: J. Appl. Phys., 66(1989) 2489.

4) J.H.Watson: J. Appl. Phys., 39(1968)3406.

5) C.P.Bean: Phys. Rev. Lett., 8(1962)250; C.P. Bean: Rev. Mod. Phys., 36(1964)31; H.London: Phys. Lett., 6(1963)162.
6) F.Irie and K.Yamafuji: J. Phys. Soc. Jpn., 23 (1967)255; I.M.Green and P.Hlawiczka: Proc. IEEE, 114(1967)1329.

7) K.Yamamoto, H.Mazaki, H.Yasuoka, T. Terashima and Y.Bando: Physica C, 192 (1992) 47.

8) K.Yamamoto, H.Mazaki, H.Yasuoka, S. Katsuyama and S.Kosuge: Phys. Rev. B, 46 (1992) 1122.

9) H.Yasuoka, T.Koga and H.Mazaki: Physica C, 235-240(1994)3195.

10) H.Mazaki, H.Yasuoka, M.Kakihana and M. Yoshimura: Proc. 1993 Powder Metallurgy World Congress (1993) p.1287.

11) H.Yasuoka, H.Mazaki, N.Koide, M.Kakihana and M.Yoshimura: Advances in Superconductivity, $\mathrm{V}(1993) 179$.

12) K.Yamamoto, H.Mazaki and H.Yasuoka: Phys. Rev. B, 47(1993)915.

13) W.I.Dunn and P.Hlawiczka: Brit. J. Appl. Phys. Ser. 2, 1(1968) 1469.

14) S.Tochihara, H.Yasuoka and H.Mazaki: Physica C, 268(1996)241.

15) S.Tochihara, H.Yasuoka, H.Mazaki, M.Komatsu and M.Nagano: J. Jpn. Soc. Powder and Powder Metallurgy, 43(1996)1065.

16) T.Ishida and H.Mazaki: J. Appl. Phys., 52(1987) 6798; H.Mazaki, K.Yamamoto, H. Yasuoka: Magnetic Susceptibility of Super-conductors and Other Spin Systems, ed. By R.A.Heain (Plenum Press, New York, (1991) p.333.

17) A.L.Fetter and P.C.Hohenberg: Phys. Rev., 159(1967)330. 